\title{
ANÁLISIS ESPACIAL DE LA ANEMIA GESTACIONAL EN EL PERÚ, 2015
}

\author{
Akram Hernández-Vásquez, ${ }^{1, a}$ Diego Azañedo ${ }^{2, b}$, Daniel A. Antiporta ${ }^{3, c}$, Sandra Cortés ${ }^{4,5, d}$
}

\begin{abstract}
RESUMEN
Objetivos. Establecer las prevalencias regionales e identificar conglomerados distritales con altas prevalencias de anemia en gestantes atendidas en los establecimientos de salud públicos del Perú en el 2015. Materiales y métodos. Se realizó un estudio ecológico de datos de gestantes con anemia, registrados en el Sistema de Información del Estado Nutricional (SIEN), que fueron atendidas en 7703 establecimientos públicos de salud durante el 2015. Se calcularon prevalencias de anemia gestacional regionales y distritales. Mediante el índice de Moran se identificaron conglomerados distritales con alta prevalencia de anemia gestacional. Resultados. Se recolectó información de 311521 gestantes, distribuidas en 1638 distritos del Perú. La prevalencia nacional de anemia fue de 24,2\% (IC 95\%: 24,0-24,3) y 30,5\% en el área rural vs. 22,0\% en el área urbana. Las regiones de Huancavelica (45,5\%; IC 95\%: 44,2-46,7), Puno (42,8\%; IC 95\%: 41,9-43,7), Pasco (38,5\%; IC 95\%: 36,9-40,0), Cusco (36,0\%; IC 95\%: 35,3-36,8) y Apurímac (32,0\%; IC 95\%: 30,8$33,1)$ tuvieron las mayores prevalencias de anemia. El índice local de Moran identificó 202 distritos (12,3\%) (44 urbanos y 158 rurales) de alta prioridad (alto-alto o hot spots) situados en Ancash, Apurímac, Arequipa, Ayacucho, Cajamarca, Cusco, Huancavelica, Huánuco, Junín, La Libertad, Lima, Pasco y Puno, que muestran conglomerados distritales con altas prevalencias. Conclusiones. La anemia gestacional en Perú concentra sus mayores prevalencias en las áreas rural y sur de la sierra. Los conglomerados distritales con altas prevalencias de anemia gestacional coinciden con las zonas de alta prevalencia regional.
\end{abstract}

Palabras clave: Sistemas de Información Geográfica; Anemia; Mujeres Embarazadas; Embarazo; Perú (fuente: DeCS BIREME).

\section{SPATIAL ANALYSIS OF GESTATIONAL ANEMIA IN PERU, 2015}

\begin{abstract}
Objectives. To establish regional prevalences of anemia in pregnant women receiving care at public clinics in Peru in 2015 and identify high-prevalence district conglomerates. Materials and Methods. An ecological study was carried out on data from pregnant women with anemia registered on the Nutritional Status Information System (SIEN) who received care in 7703 public clinics in 2015. Regional and district prevalences of gestational anemia were calculated. District conglomerates with a high prevalence of gestational anemia were identified using the Moran Index. Results. Information was gathered from 311,521 pregnant women distributed in 1638 districts in Peru. The national prevalence of anemia was $24.2 \%$ (95\% confidence interval [95\% Cl]: $24.0-24.3 \%)$, the rural prevalence was $30.5 \%$, and the urban prevalence was $22.0 \%$. The regions of Huancavelica (45.5\%; 95\% Cl: 44.2-46.7\%), Puno (42.8\%; 95\% Cl: 41.9-43.7\%), Pasco (38.5\%; 95\% Cl: 36.9-40.0\%), Cusco (36.0\%; 95\% Cl: 35.3-36.8\%), and Apurímac (32.0\%; 95\% Cl: 30.8-33.1\%) had the highest prevalences of anemia. The local Moran Index identified 202 high-priority districts (hot spots) (12.3\% of total; 44 urban and 158 rural) located in Ancash, Apurímac, Arequipa, Ayacucho, Cajamarca, Cusco, Huancavelica, Huánuco, Junín, La Libertad, Lima, Pasco, and Puno containing high-prevalence district conglomerates. Conclusions. Gestational anemia in Peru has its highest prevalence rates in rural and southern mountainous areas. The district conglomerates with high prevalence rates of gestational anemia coincide with the areas of high regional prevalence.
\end{abstract}

Key words: Geographic Information Systems; Anemia; Pregnant Women; Pregnancy; Peru (source: MeSH NLM).

Universidad de Buenos Aires. Buenos Aires, Argentina.

Instituto de Investigación, Universidad Católica los Ángeles de Chimbote. Chimbote, Perú.

Johns Hopkins Bloomberg School of Public Health. Baltimore, EE. UU.

Facultad de Medicina, Pontificia Universidad Católica de Chile. Santiago, Chile.

Advanced Center for Chronic Diseases (ACCDiS). Santiago, Chile.

Médico cirujano, magíster en Gestión y Políticas Públicas; ${ }^{\mathrm{b}}$ cirujano dentista, ${ }^{\mathrm{c}}$ licenciado en nutrición, magíster en Salud Pública ${ }^{\mathrm{d}}$ médico veterinario, doctora en Salud Pública.

Recibido: 14/06/2016 Aprobado:11/01/2017 En línea: 23/03/2017

Citar como: Hernández-Vásquez A, Azañedo D, Antiporta DA, Cortés S. Análisis espacial de la anemia gestacional en el Perú, 2015. Rev Peru Med Exp Salud Publica. 2017;34(1):43-51. doi:10.17843/rpmesp.2017.341.2707 


\section{INTRODUCCIÓN}

La anemia es una alteración frecuente durante el embarazo y su asociación con un mayor riesgo de resultados adversos sobre la morbimortalidad materna y neonatal la convierten en un problema de salud pública ${ }^{(1,2)}$. A nivel mundial, se han generado avances en la reducción de la prevalencia de anemia gestacional, pasando de $43 \%$ en 1995 a 38\% en 2011, y de 37 a 31\% en países de América Latina y el Caribe en el mismo periodo ${ }^{(3)}$. A pesar de esta disminución, aún representa un importante problema de salud en los países en desarrollo ${ }^{(4)}$ y contribuye con el $20 \%$ de todas las muertes maternas en todo el mundo ${ }^{(5)}$.

En el Perú también se ha observado una reducción en la prevalencia de anemia gestacional, que va de $42,7 \%$ en el $2004{ }^{(6)}$, hasta $28,0 \%$ en el $2011^{(7)}$. A pesar de este avance, aún continúa como un problema de salud pública de moderada intensidad, según la clasificación de la Organización Mundial de la Salud ${ }^{(8)}$. Además, se debe tener en cuenta que la distribución de esta enfermedad responde a múltiples factores, encontrándose fuertemente asociada a variables sociodemográficas y territoriales ${ }^{(9-11)}$ que, en un país tan diverso como el Perú, confluyen para generar marcadas disparidades y el mantenimiento de zonas con altas prevalencias de anemia gestacional. En tal sentido, la identificación de patrones de distribución territorial de esta enfermedad puede ser útil para identificar zonas de mayor concentración en donde se puedan proveer mayores cuidados materno-perinatales, así como la identificación y cuantificación de otras condiciones sociodemográficas y ambientales asociadas a la anemia.

Los sistemas de información geográfica (SIG) han ganado atención por sus aplicaciones en diversos problemas de salud pública, permitiendo un mejor abordaje y facilitando el estudio de su asociación con diversas características sociodemográficas poblacionales ${ }^{(12-14)}$. Los SIG pueden ser aplicados en el análisis de la distribución geoespacial de la anemia gestacional, y ser una herramienta de ayuda para la implementación de acciones preventivo-promocionales, así como en el diseño y seguimiento de programas y políticas en salud orientadas a mejorar la salud materna. Una metodología aplicada dentro de este tipo de análisis es el índice de Moran, el cual, mediante la evaluación de la correlación espacial, permite determinar la presencia de conglomerados poblacionales donde la necesidad de abordar los problemas de salud sea prioritaria ${ }^{(15)}$. Sin embargo, en el Perú, la utilización de estos sistemas es muy limitada y aún no han sido lo suficientemente usados para el estudio del problema de la anemia gestacional.
El objetivo de este estudio fue establecer prevalencias regionales e identificar conglomerados distritales con altas prevalencias de anemia en gestantes atendidas en los establecimientos de salud públicos del Perú.

\section{MATERIALES Y MÉTODOS}

\section{DISEÑO Y ÁMBITO DEL ESTUDIO}

Se realizó un estudio de tipo ecológico de comparación geográfica a partir de las prevalencias de anemia en 311 521 gestantes que acudieron a 7703 establecimientos públicos del Perú para sus controles prenatales, entre el 1 de enero al 31 de diciembre de 2015.

Se evaluó la información de las gestantes teniendo como unidad de análisis las regiones y distritos de residencia. Cabe precisar que, en el Perú, luego de un proceso de descentralización, su territorio quedó dividido geopolíticamente en 25 regiones y 1845 distritos que se encuentran distribuidos en tres regiones naturales: la costa con su vecindad al océano Pacífico que alberga la mayor población urbana, la sierra que circunscribe a la cordillera de los Andes y la selva conformada por la Amazonía peruana.

\section{FUENTES DE INFORMACIÓN}

La información de gestantes controladas y casos de anemia detectados fueron obtenidos del Sistema de Información del Estado Nutricional (SIEN) (16), elaborada por el Instituto Nacional de Salud (INS) que tiene como finalidad el registrar, procesar, reportar y analizar información del estado nutricional de las gestantes que acuden a los establecimientos públicos de salud en 1845 distritos del Perú. Las prevalencias de anemia como problema de salud pública fueron clasificados teniendo en cuenta los criterios establecidos por la Organización Mundial de la Salud (OMS): $<5 \%$, no representa un problema de salud pública; 5 a $19,9 \%$, problema de salud pública leve; 20 a $39,9 \%$, problema de salud pública moderado, $y \geq 40 \%$, se considera como problema de salud pública grave. El Decreto Supremo 090-2011-PCM fue usado como segunda fuente para categorizar a los distritos incluidos en el SIEN como urbanos o rurales. La última fuente de información consiste en una base cartográfica que contiene los 1834 distritos existentes en el Perú para el año 2007, elaborada por el Instituto Nacional de Estadística e Informática (INEI).

\section{ANÁLISIS DE DATOS}

Se realizó un análisis descriptivo y transversal, mediante el software estadístico Stata 14.1 (StataCorp LP, College Station, TX, USA), para determinar prevalencias de anemia según regiones naturales y área de residencia 
(urbana/rural), y razones de prevalencia (RP) entre áreas urbanas y rurales y regiones naturales, con intervalos de confianza al 95\%; así como también, para cada una de las 25 regiones y los 1834 distritos del Perú. Las tasas distritales obtenidas fueron incluidas en una hoja de cálculo de Microsoft Excel® 2013 y una investigadora externa verificó que los datos incluidos sean los correctos.

La determinación de agrupaciones distritales por altas tasas de anemia se realizó mediante un análisis espacial usando el software GeoDa 1.6.7 (GeoDa Center for Geospatial Analysis and Computation, Arizona State University, Tempe, AZ, USA), partiendo de la integración de la hoja de cálculo a la base cartográfica que obtuvo una capa resultante de prevalencias de anemia en gestantes de 1834 distritos (Figura 1). Para explorar la autocorrelación espacial distrital a nivel nacional, se utilizó el índice global y local de Moran con un nivel de significancia de 0,05 utilizando 999 permutaciones, que miden la tendencia de las unidades estudiadas a agruparse espacialmente. El cálculo del índice global de Moran se inicia con la asignación de una matriz de pesos geográficos basado en la relación entre las unidades estudiadas mediante contigüidad 1 a 1 tipo Queen. La visualización de la correlación se realiza mediante un diagrama de dispersión con valores que van desde -1 $a+1$, permitiendo evaluar si las unidades de análisis presentan tendencia a la agrupación, dispersión o aleatorización. Siendo indicativo de agrupación espacial (autocorrelación positiva) aquellos valores mayores a cero, de una distribución aleatorizada para los valores iguales a cero y los valores menores a cero indican una dispersión espacial (autocorrelación negativa) de las unidades de análisis. La representación espacial se realizó mediante el índice local de Moran conocido como LISA, el cual representa cinco tipos de agrupamiento o conglomerados: 1) conglomerados distritales con altas prevalencias de anemia rodeados por distritos con prevalencia superior a la media "alto-alto" o hot spots; 2) conglomerados distritales con altas prevalencias rodeados por distritos con prevalencias bajo el promedio "alto-bajo"; 3) conglomerados distritales con bajas prevalencias rodeados por distritos con prevalencia que superan la media "bajo-alto"; 4) conglomerados con bajas prevalencias distritales de anemia rodeados por distritos con bajas prevalencias "bajo-bajo", y 5) distritos con prevalencias que no se correlacionan con los distritos que los rodean "no significativas". Se usó el programa ArcGIS Desktop versión 10.4 (ESRI Inc., Redlands, CA, USA), para representar un mapa de prevalencias según clasificación de la OMS y un mapa de conglomerados.

Como paso final, los resultados del análisis espacial con las agrupaciones distritales alto-alto fueron exportados a una hoja de cálculo para clasificarlos según su categoría de urbano o rural y por regiones.

\section{CONSIDERACIONES ÉTICAS}

La realización del estudio no requirió la aprobación de un comité de ética por tratarse de un análisis de datos secundarios agregados obtenidos de un dominio público y de libre acceso, que no permite identificar bajo ninguna circunstancia a las gestantes evaluadas.

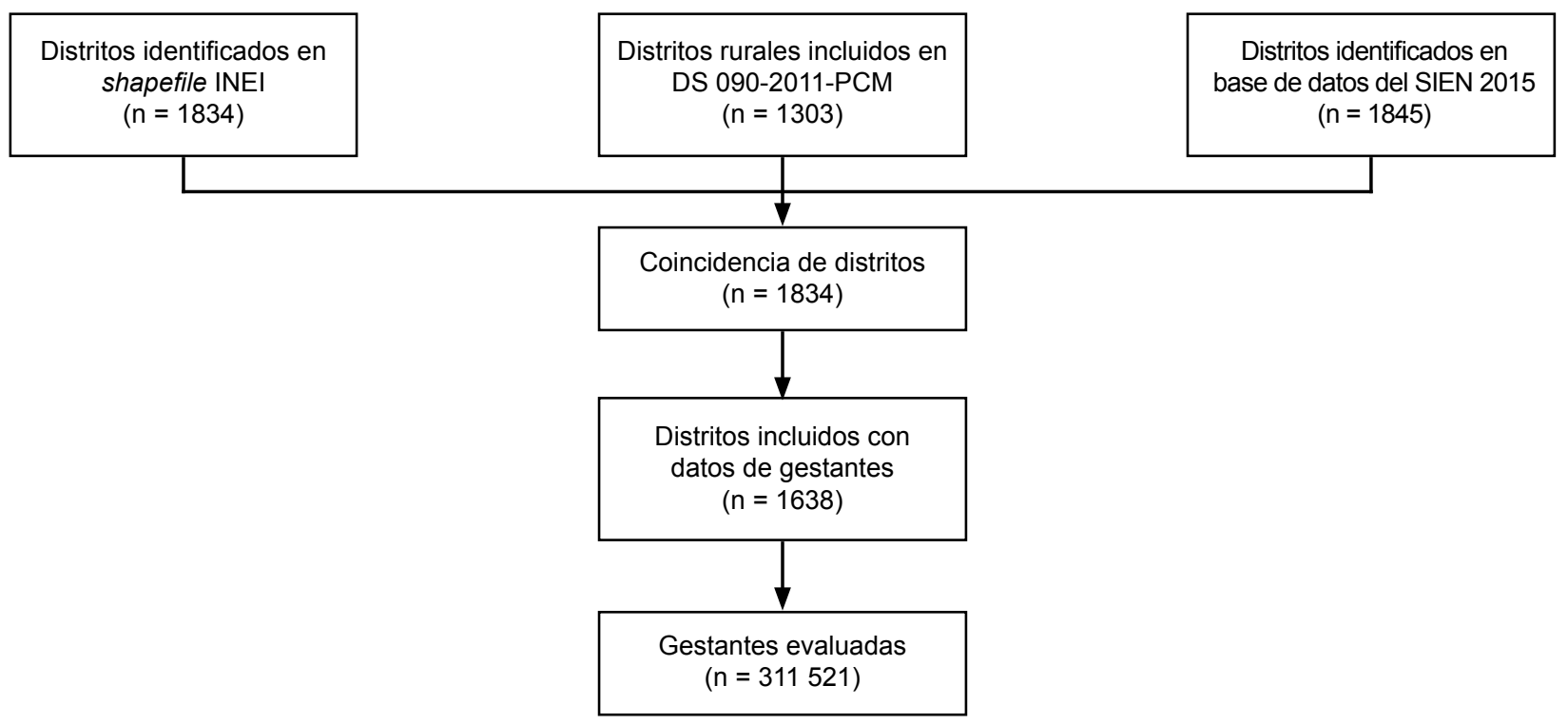

Figura 1. Elaboración de la base cartográfica distrital 


\section{RESULTADOS}

Se identificaron datos de gestantes evaluadas en 1638 distritos de un total de 1834. Durante el año de estudio se evaluaron 311521 gestantes, de las cuales 75307 se encontraban con anemia, siendo la prevalencia nacional de 24,2\% (IC 95\%: 24,0-24,3). Las mayores prevalencias de anemia se identificaron en zonas andinas de las regiones del sur del Perú. Asimismo, se hallaron prevalencias distritales con rango entre $0 \%$ (51 distritos) y $100 \%$ (8 distritos). Según la clasificación de la prevalencia de anemia como problema de salud pública establecida por la OMS, en 463 distritos es un grave problema de salud pública (prevalencia $\geq 40 \%$ ) y en 601 distritos es un moderado problema de salud pública (prevalencia de $20 \%$ a 39,9\%) (Figura 2). En 888 distritos la prevalencia de anemia gestacional está por encima del promedio nacional $(24,2 \%)$.
La prevalencia de anemia gestacional fue mayor en el área de residencia rural $(30,5 \%)$ y en la región natural de la sierra $(30,7 \%)$. De igual manera, al comparar entre zonas rurales y urbanas de cada región natural, se presentaron los más altos porcentajes en las áreas rurales de la costa $(29,9 \%)$, sierra $(32,8 \%)$ y selva peruana $(22,5 \%)$ (Tabla 1$)$.

El análisis espacial mostró un índice global de Moran para las prevalencias distritales de anemia gestacional de 0,28 $(p=0,001)$. De los 1638 distritos evaluados, el mapa del índice local de Moran identificó 202 (12,33\%) distritos (44 urbanos y 158 rurales) de alta prioridad ("alto-alto o hot spots") situados en Ancash, Apurímac, Arequipa, Ayacucho, Cajamarca, Cusco, Huancavelica, Huánuco, Junín, La Libertad, Lima, Pasco y Puno, que muestran conglomerados distritales con altas prevalencias, rodeados de distritos con prevalencias sobre el promedio (Figura 3).

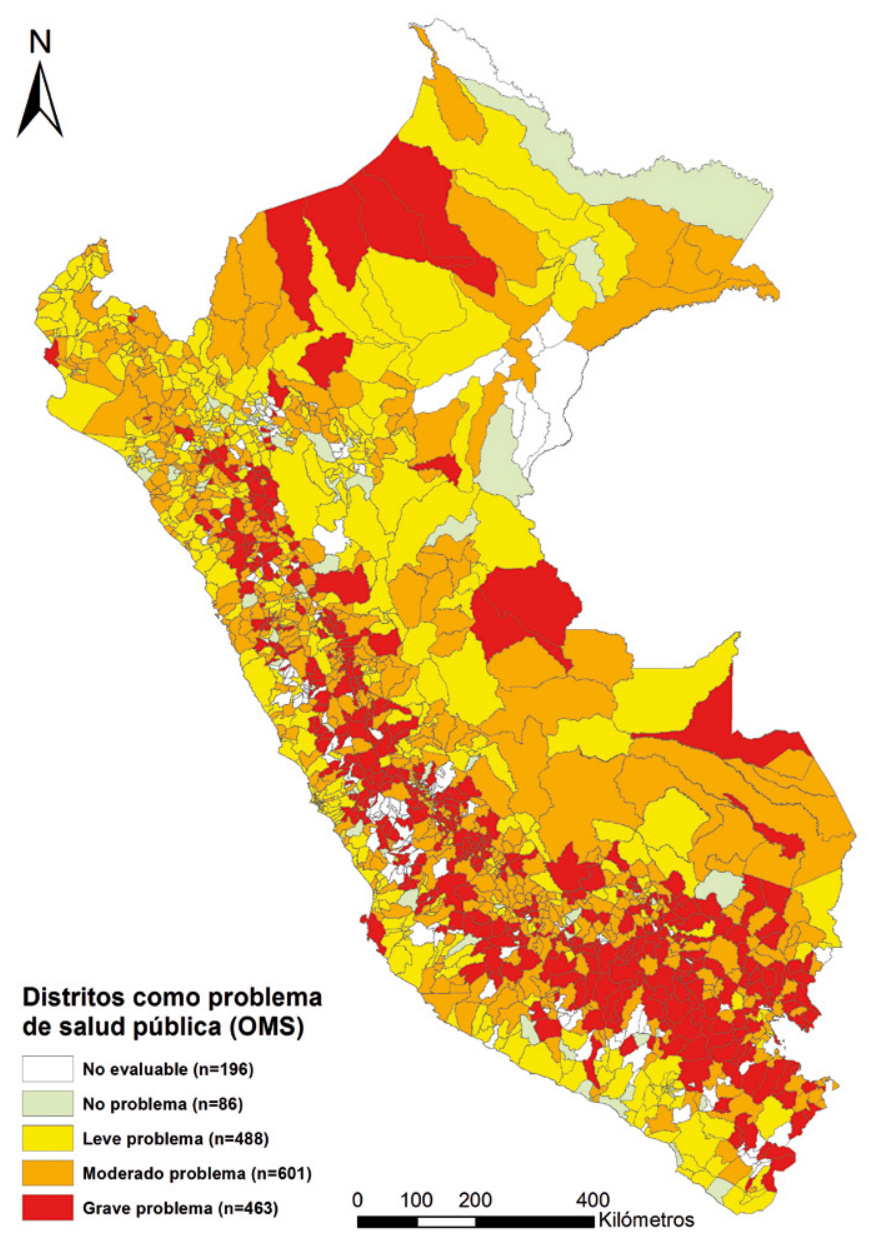

Figura 2. Distritos con anemia gestacional como problema de salud pública, Perú 2015 
Tabla 1. Prevalencias de anemia gestacional según regiones naturales y área de residencia, Perú 2015

\begin{tabular}{|c|c|c|c|c|c|c|}
\hline \multirow{2}{*}{ Características } & \multirow{2}{*}{ Número de gestantes evaluadas } & \multicolumn{3}{|c|}{ Casos detectados } & \multirow[b]{2}{*}{ RP (IC 95\%) } & \multirow[b]{2}{*}{ Valor $\mathrm{p}$} \\
\hline & & $\mathbf{n}$ & $\%$ & (IC 95\%) & & \\
\hline \multicolumn{7}{|c|}{ Área de residencia } \\
\hline Urbana & 230373 & 50586 & 22,0 & $(21,8-22,1)$ & 1 & \\
\hline Rural & 81148 & 24721 & 30,5 & $(30,1-30,8)$ & $1,39(1,36-1,41)$ & $<0,001$ \\
\hline \multicolumn{7}{|l|}{ Región natural } \\
\hline Costa & 144456 & 28841 & 20,0 & $(19,8-20,2)$ & 1 & \\
\hline Sierra & 123822 & 37971 & 30,7 & $(30,4-30,9)$ & $1,54(1,51-1,56)$ & $<0,001$ \\
\hline Selva & 43243 & 8495 & 19,6 & $(19,3-20,0)$ & $0,98(0,96-1,00)$ & 0,023 \\
\hline \multicolumn{7}{|l|}{ Costa } \\
\hline Urbana & 130756 & 24744 & 18,9 & $(18,7-19,1)$ & 1 & \\
\hline Rural & 13700 & 4097 & 29,9 & $(29,1-30,7)$ & $1,58(1,52-1,64)$ & $<0,001$ \\
\hline \multicolumn{7}{|l|}{ Sierra } \\
\hline Urbana & 72708 & 21022 & 28,9 & $(28,6-29,2)$ & 1 & \\
\hline Rural & 51114 & 16949 & 32,8 & $(32,8-33,6)$ & $1,15(1,12-1,17)$ & $<0,001$ \\
\hline \multicolumn{7}{|l|}{ Selva } \\
\hline Urbana & 26909 & 4828 & 17,9 & $(17,5-18,4)$ & 1 & \\
\hline Rural & 16334 & 3675 & 22,5 & $(21,9-23,1)$ & $1,25(1,20-1,31)$ & $<0,001$ \\
\hline
\end{tabular}

Del total de regiones, Huancavelica $(45,5 \%)$, Puno $(42,8 \%)$, Pasco $(38,5 \%)$, Cusco $(36,0 \%)$ y Apurímac $(32,0 \%)$, fueron las que tuvieron mayor número de casos detectados de anemia en las gestantes evaluadas. En todos los casos anteriores el número de distritos afectados es superior al $89,4 \%$ del total de distritos

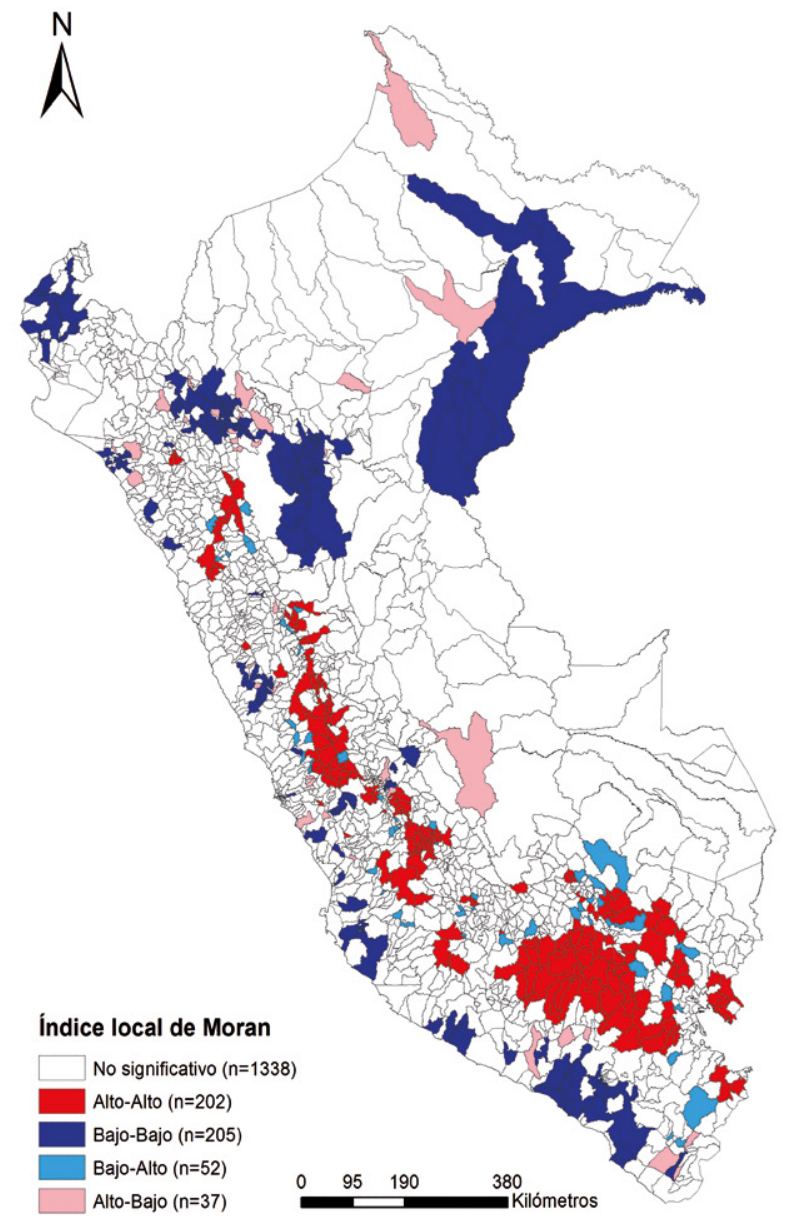

Figura 3. Análisis espacial de las prevalencias de anemia en gestantes, Perú 2015. Los conglomerados según el índice local de Moran fueron alto-alto (rojo) concentran distritos con altas prevalencias de anemia y los bajo-bajo (azul) concentran distritos con baja prevalencia. 
que posee cada región según la fuente consultada. La anemia gestacional representa un problema de salud pública entre moderado y grave en 1064 (65,0\%) del total de distritos evaluados (Tabla 2).

De los 202 distritos identificados en conglomerados hot spots, el $78,2 \%$ eran rurales. Se observó distritos en conglomerados de anemia gestacional en 13 regiones. Pasco (35,7\%), Cusco (33,3\%), Huancavelica (31,9\%), Puno $(24,8 \%)$ y Junín $(17,9 \%)$ reportaron la mayor cantidad de distritos ubicados en conglomerados de altas prevalencias de anemia, en relación al total de distritos que conforman dichas regiones (Tabla 3).

Tabla 2. Prevalencia de anemia gestacional según regiones y clasificación de distritos como problema de salud pública, Perú 2015

\begin{tabular}{|c|c|c|c|c|c|c|c|}
\hline \multirow{2}{*}{ Regiones } & \multirow{2}{*}{$\begin{array}{l}\text { Número de } \\
\text { gestantes } \\
\text { evaluadas }\end{array}$} & \multicolumn{3}{|c|}{ Casos detectados } & \multicolumn{3}{|c|}{$\begin{array}{l}\text { Distritos como problema de salud } \\
\text { pública* }\end{array}$} \\
\hline & & $\mathbf{n}$ & $\%$ & (IC 95\%) & $\begin{array}{c}\text { Total } \\
\text { distritos }\end{array}$ & $\begin{array}{c}\text { Problema } \\
\text { moderado } \\
(\%)\end{array}$ & $\begin{array}{l}\text { Problema } \\
\text { grave }(\%)\end{array}$ \\
\hline Amazonas & 8235 & 1688 & 20,5 & $(19,6-21,4)$ & 51 & $16(31,4)$ & $5(9,8)$ \\
\hline Ancash & 11452 & 3401 & 29,7 & $(28,9-30,5)$ & 127 & $55(43,3)$ & $43(33,9)$ \\
\hline Apurímac & 6494 & 2076 & 32,0 & $(30,8-33,1)$ & 72 & $39(54,2)$ & $28(38,9)$ \\
\hline Arequipa & 14953 & 2239 & 15,0 & $(14,4-15,6)$ & 81 & $18(22,2)$ & $21(25,9)$ \\
\hline Ayacucho & 11803 & 3779 & 32,0 & $(31,2-32,9)$ & 99 & $52(52,5)$ & $32(32,3)$ \\
\hline Cajamarca & 17116 & 4291 & 25,1 & $(24,4-25,7)$ & 113 & $46(40,7)$ & $21(18,6)$ \\
\hline Callao & 5986 & 954 & 15,9 & $(15,0-16,9)$ & 6 & $(0,0)$ & $0(0,0)$ \\
\hline Cusco & 15104 & 5441 & 36,0 & $(35,3-36,8)$ & 103 & $32(31,1)$ & $53(51,5)$ \\
\hline Huancavelica & 6147 & 2795 & 45,5 & $(44,2-46,7)$ & 84 & $31(36,9)$ & $44(52,4)$ \\
\hline Huánuco & 13706 & 3749 & 27,4 & $(26,6-28,1)$ & 76 & $31(40,8)$ & $26(34,2)$ \\
\hline Ica & 9681 & 1799 & 18,6 & $(17,8-19,4)$ & 37 & $10(27,0)$ & $1(2,7)$ \\
\hline Junín & 10603 & 3330 & 31,4 & $(30,5-32,3)$ & 101 & $38(37,6)$ & $45(44,6)$ \\
\hline La Libertad & 16959 & 4930 & 29,1 & $(28,4-29,8)$ & 78 & $39(50,0)$ & $21(26,9)$ \\
\hline Lambayeque & 12016 & 1924 & 16,0 & $(15,4-16,7)$ & 31 & $12(38,7)$ & $1(3,2)$ \\
\hline Lima & 74763 & 13999 & 18,7 & $(18,4-19,0)$ & 130 & $35(26,9)$ & $38(29,2)$ \\
\hline Loreto & 14542 & 2648 & 18,2 & $(17,6-18,8)$ & 39 & $15(38,5)$ & $6(15,4)$ \\
\hline Madre de Dios & 2269 & 695 & 30,6 & $(28,7-32,6)$ & 11 & $8(72,7)$ & $2(18,2)$ \\
\hline Moquegua & 2220 & 344 & 15,5 & $(14,0-17,1)$ & 14 & $6(42,9)$ & $3(21,4)$ \\
\hline Pasco & 3871 & 1489 & 38,5 & $(36,9-40,0)$ & 27 & $10(37,0)$ & $11(40,7)$ \\
\hline Piura & 15636 & 3291 & 21,0 & $(20,4-21,7)$ & 60 & $25(41,7)$ & $2(3,3)$ \\
\hline Puno & 12573 & 5381 & 42,8 & $(41,9-43,7)$ & 104 & $46(44,2)$ & $49(47,1)$ \\
\hline San Martín & 12007 & 2136 & 17,8 & $(17,1-18,5)$ & 62 & $19(30,6)$ & $1(1,6)$ \\
\hline Tacna & 2724 & 525 & 19,3 & $(17,8-20,8)$ & 18 & $3(16,7)$ & $8(44,4)$ \\
\hline Tumbes & 4471 & 1075 & 24,0 & $(22,8-25,3)$ & 13 & $6(46,2)$ & $0(0,0)$ \\
\hline Ucayali & 6190 & 1328 & 21,5 & $(20,4-22,5)$ & 15 & $9(60,0)$ & $2(13,3)$ \\
\hline
\end{tabular}

*Según clasificación de la Organización Mundial de la Salud (OMS) 
Tabla 3. Distritos con alta prevalencia de anemia identificados en los conglomerados de análisis espacial según regiones naturales y área de residencia, Perú 2015

\begin{tabular}{lrcr}
\hline \multirow{2}{*}{ Regiones } & $\begin{array}{c}\text { Total de } \\
\text { distritos }\end{array}$ & \multicolumn{2}{c}{$\begin{array}{c}\text { Conglomerados de } \\
\text { anemia gestacional }\end{array}$} \\
\cline { 2 - 4 } & & Urbano & Rural \\
\hline Amazonas & 84 & $\mathrm{NI}$ & $\mathrm{NI}$ \\
Ancash & 166 & $\mathrm{NI}$ & 8 \\
Apurímac & 80 & $\mathrm{NI}$ & 8 \\
\hline Arequipa & 109 & 2 & 15 \\
Ayacucho & 111 & 2 & 8 \\
Cajamarca & 127 & $\mathrm{NI}$ & 4 \\
Callao & 6 & $\mathrm{NI}$ & $\mathrm{NI}$ \\
Cusco & 108 & 7 & 29 \\
\hline Huancavelica & 94 & 7 & 23 \\
Huánuco & 76 & $\mathrm{NI}$ & 13 \\
\hline Ica & 43 & $\mathrm{NI}$ & $\mathrm{NI}$ \\
Junín & 123 & 8 & 14 \\
La Libertad & 83 & 2 & 7 \\
Lambayeque & 38 & $\mathrm{NI}$ & $\mathrm{NI}$ \\
Lima & 171 & 2 & 6 \\
\hline Loreto & 51 & $\mathrm{NI}$ & $\mathrm{NI}$ \\
\hline Madre de Dios & 11 & $\mathrm{NI}$ & $\mathrm{NI}$ \\
\hline Moquegua & 20 & $\mathrm{NI}$ & 1 \\
\hline Pasco & 28 & 7 & 3 \\
\hline Piura & 64 & $\mathrm{NI}$ & $\mathrm{NI}$ \\
\hline Puno & 109 & 7 & 20 \\
\hline San Martín & 77 & $\mathrm{NI}$ & $\mathrm{NI}$ \\
\hline Tacna & 27 & $\mathrm{NI}$ & $\mathrm{NI}$ \\
\hline Tumbes & 13 & $\mathrm{NI}$ & $\mathrm{NI}$ \\
\hline Ucayali & 15 & $\mathrm{NI}$ & $\mathrm{NI}$ \\
\hline & & & \\
\hline
\end{tabular}

$\mathrm{NI}=$ No identificados

\section{DISCUSIÓN}

El presente estudio identificó regiones y conglomerados distritales con altas prevalencias de anemia gestacional, concentrados, mayormente, en la parte central y sur de la sierra peruana. La prevalencia nacional de anemia en gestantes fue de $24,2 \%$, asimismo las regiones con mayor prevalencia fueron: Huancavelica, Puno, Pasco, Cusco y Apurímac. El mayor número de gestantes con anemia se concentró en las áreas de residencia rurales de las tres regiones naturales del Perú. Según criterio de la OMS la presencia de anemia representa un grave problema de salud pública en 463 distritos y moderado problema de salud pública en 601 distritos peruanos. Los 202 distritos identificados en conglomerados hot spots se distribuyeron en 13 regiones y el mayor porcentaje de estos distritos eran rurales.

Respecto a la prevalencia nacional de anemia gestacional identificada, nuestras cifras se encuentran ligeramente por debajo de las reportadas en Brasil (32\%) Argentina (28\%), Chile (25\%), Uruguay (29\%), Ecuador
(29\%) y Colombia (30\%); a pesar de esta posición favorecida con respecto a nuestros países vecinos la brecha se amplía cuando hacemos comparaciones con países desarrollados, como Estados Unidos (17\%), Reino Unido (23\%) y Canadá $(23 \%)$ así como otros de similar desarrollo: Nicaragua (20\%) y México $(21 \%)$, estos últimos deberían ser objeto de estudio para la identificación de factores que permitieron lograr una disminución de sus prevalencias de anemia en gestantes por debajo de la media de Latinoamérica y el Caribe $(28,2 \%)^{(17)}$.

Asimismo, los patrones de concentración de anemia gestacional de nuestro estudio demuestran un predominio rural, el cual es un sector donde los componentes educación, nutrición, deterioro ambiental, pobreza y economía pueden determinar la presencia de múltiples problemas de salud, incluida la anemia ${ }^{(18)}$. En nuestro estudio, la diferencia porcentual de las prevalencias urbanas y rurales es de más de ocho puntos, no obstante, en otros contextos, como el de México, esta no presenta diferencias en sus proporciones según áreas de residencia, por lo que sería interesante, estudiar esta característica, y comparar resultados con Perú (19). En contraste con nuestros hallazgos, un estudio en Haití reportó que la mayor parte de la población de mujeres anémicas en edad gestacional vivía en áreas urbanas, respondiendo a diferentes factores que en las comunidades rurales (quintil de ingresos y empleo), viéndose asociados en cambio, con el nivel educativo y sobrepeso ${ }^{(20)}$. Esto de alguna manera nos permite inferir que son necesarias estrategias específicas para diversas áreas de la población, más aun en aquellas donde el área de residencia puede ser un factor determinante para la presencia de múltiples enfermedades.

Por otro lado, se observa variabilidad en las prevalencias de anemia materna entre regiones naturales del país, siendo mayores en la sierra, donde coinciden la mayor cantidad de áreas rurales nacionales. Las variaciones de la prevalencia de anemia dentro de una misma área geográfica responden a factores de riesgo, socioeconómicos y demográficos ${ }^{(21-23)}$, que en Perú presentan marcada variación en su distribución y que estarían contribuyendo a generar diversos patrones de concentración de anemia gestacional en las diferentes regiones. Por otro lado, la literatura menciona la implicancia de la variación geográfica en la detección de casos de anemia, pues algunos autores sugieren que específicamente aquellas regiones de mayor altitud podrían influir negativamente en el diagnóstico de anemia, en vista de que los parámetros utilizados actualmente no son los recomendados por la Organización Mundial de la Salud para las zonas de mayor altura ${ }^{(24,25)}$, en ese caso se considera que al ajustar dichos parámetros los resultados en zonas de altura que podrían incrementar la prevalencia. 
Cabe resaltar que, a pesar de la disminución de la prevalencia de anemia a nivel nacional, aún hay zonas donde se mantienen elevadas al punto de ser consideradas como grave problema de salud pública, tal es el caso de Huancavelica, Puno, Pasco, Cusco y Apurímac, cuyas prevalencias son las más altas de

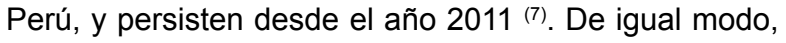
el análisis de índice de Morán identificó la presencia de conglomerados distritales de altas prevalencias, ubicándose, mayormente, en la zona centro y sur de la sierra, tendencia similar a la reportada por un estudio el 2011, que identificó las mayores prevalencias en regiones del sur andino ${ }^{(26)}$.

Tomando en cuenta los hallazgos, y la existencia de estrategias preventivas que en otros países han demostrado costo-efectividad en la disminución de casos de anemia ${ }^{(27,28)}$, la presencia de esta enfermedad en algunas regiones y distritos peruanos, no serían un problema de la disponibilidad de medidas preventivas, sino en parte de una inadecuada focalización de esfuerzos que buscan su utilización, debiendo enfatizarse en las zonas de mayor prioridad. Para lograr esto último, el uso de sistemas de evaluación, información y vigilancia con un mayor nivel de precisión y visualización, como el monitoreo a nivel geográfico, son cruciales.

Existen algunas limitaciones relacionadas con la utilización de la base de datos del SIEN, por lo que no podemos garantizar que se realizó un correcto llenado de la misma, ni en la especificidad y sensibilidad de las pruebas utilizadas para el diagnóstico de anemia. Sin embargo, la fuente utilizada es representativa, por lo que permite realizar inferencias a nivel regional y nacional por el número de gestantes atendidas en los establecimientos de salud públicos, los cuales se distribuyen ampliamente por el territorio peruano. Los resultados reportados pueden servir para observar los patrones de prevalencia y distribución regional de la anemia gestacional en la actualidad, y comparar con los de estudios pasados o futuros, para evaluar avances en este aspecto, además va a permitir a los tomadores de decisiones mejorar la disposición, distribución y promoción de estrategias contra la anemia en el Perú. De igual manera, nuestro estudio fue el primero en utilizar un nivel distrital de análisis geográfico que permitió observar conglomerados de alta prevalencia de anemia gestacional en el Perú.

En conclusión, la anemia gestacional sigue siendo un moderado problema de salud pública a nivel nacional; asimismo, se identificaron las mayores prevalencias regionales de anemia gestacional en el área rural y la parte sur de la sierra peruana, representando un grave problema de salud pública en la mayor parte de ellas, además, el análisis geoespacial ubicó conglomerados distritales con altas prevalencias de anemia gestacional distribuidos en trece regiones del país, que coinciden con las zonas de alta prevalencia regional. El análisis geoespacial utilizando SIG, permite una mejor visualización de los problemas de salud, facilitando la toma de decisiones y logrando focalizar las estrategias de salud pública en los distritos y regiones con mayor necesidad. La existencia de estos hallazgos requiere de la respuesta efectiva e inmediata de medidas preventivopromocionales que refuercen el cuidado de las mujeres embarazadas con el objetivo de reducir los altos índices de anemia en las zonas geográficas de alta prioridad.

Contribuciones de autoría: AHV tuvo la idea de investigación. AHV y DA diseñaron el estudio y recopilaron los datos. AHV realizó el procesamiento espacial de los datos. AHV, DA, DAA y SC analizaron e interpretaron los datos, redactaron el manuscrito y aprobaron la versión final.

Conflictos de interés: los autores declaran no tener conflictos de interés.

Fuentes de financiamiento: autofinanciado.

\section{REFERENCIAS BIBLIOGRÁFICAS}

1. World Health Organization (WHO). The global prevalence of anemia in 2011 [Internet]. Geneva, Switzerland: WHO; 2011 [citado el 30 de marzo de 2016]. Disponible en: http://apps.who.int/iris/bitstre $\mathrm{am} / 10665 / 177094 / 1 / 9789241564960$ eng.pdf.

2. Allen LH. Anemia and iron deficiency: effects on pregnancy outcome. Am J Clin Nutr. 2000 May;71(5 Suppl):1280S-4S.

3. Stevens GA, Finucane MM, De-Regil LM, Paciorek CJ, Flaxman SR, Branca
F, et al. Global, regional, and national trends in haemoglobin concentration and prevalence of total and severe anaemia in children and pregnant and non-pregnant women for 1995-2011: a systematic analysis of populationrepresentative data. Lancet Glob Health. 2013 Jul;1(1):e16-25. doi: 10.1016/S2214-109X(13)70001-9.

4. Rahman MM, Abe SK, Rahman MS, Kanda M, Narita S, Bilano V, et al. Maternal anemia and risk of adverse birth and health outcomes in low- and middle-income countries: systematic review and meta-analysis. Am J Clin Nutr. 2016 Feb;103(2):495-504. doi: 10.3945/ajcn.115.107896.

5. World Health Organization (WHO). Iron deficiency anemia [Internet]. Geneva, Switzerland: WHO; 2016 [citado el 01 de abril de 2016]. Disponible en: http://www.who.int/ nutrition/topics/ida/en/.

6. World Health Organization (WHO). Worldwide prevalence of anaemia 1993-2005 [Internet]. Ge- 
neva, Switzerland: WHO; 2008 [citado el 28 de marzo de 2016]. Disponible en: http://apps.who.int/iris/bitstre am/10665/43894/1/9789241596657_ eng.pdf.

7. Munares-Garcia O, Gomez-Guizado G, Barboza-Del Carpio J, SanchezAbanto J. [Hemoglobin levels in pregnant women seen in health care centers of the Peruvian Ministry of Health, 2011]. Rev Peru Med Exp Salud Publica. 2012;29(3):329-36.

8. Mujica-Coopman MF, Brito A, Lopez de Romana D, Rios-Castillo I, Coris $\mathrm{H}$, Olivares M. Prevalence of Anemia in Latin America and the Caribbean. Food Nutr Bull. 2015 Jun;36(2 Suppl):S119-28.

9. Haniff J, Das A, Onn LT, Sun CW, Nordin NM, Rampal S, et al. Anemia in pregnancy in Malaysia: a crosssectional survey. Asia Pac J Clin Nutr. 2007;16(3):527-36.

10. Pei L, Ren L, Wang D, Yan $\mathrm{H}$. Assessment of maternal anemia in rural Western China between 2001 and 2005: a two-level logistic regression approach. BMC Public Health. 2013 Apr 19;13:366. doi: 10.1186/14712458-13-366.

11. Noronha JA, Bhaduri A, Vinod Bhat $\mathrm{H}$, Kamath A. Maternal risk factors and anaemia in pregnancy: a prospective retrospective cohort study. J Obstet Gynaecol. 2010 Feb;30(2):132-6. doi: $10.3109 / 01443610903267457$.

12. Eccles KM, Bertazzon S. Applications of geographic information systems in public health: A geospatial approach to analyzing MMR immunization uptake in Alberta. Can J Public Health. 2015 Jun 18;106(6):e355-61. doi: 10.17269/cjph.106.4981.

13. Kaminska IA, Oldak A, Turski WA. Geographical Information System (GIS) as a tool for monitoring and analysing pesticide pollution and its impact on public health. Ann Agric Environ Med. 2004;11(2):181-4.

14. Chong S, Nelson M, Byun R, Harris L, Eastwood J, Jalaludin B. Geospatial analyses to identify clusters of adverse antenatal factors for targeted interventions. Int J Health Geogr. 2013 Oct 24;12:46. doi: 10.1186/1476072X-12-46.

15. Stopka TJ, Krawczyk C, Gradziel P, Geraghty EM. Use of Spatial Epidemiology and Hot Spot Analysis to Target Women Eligible for Prenatal Women, Infants, and Children Services. Am J Public Health. 2014 Feb;104 Suppl 1:S183-9. doi: 10.2105/ AJPH.2013.301769.

16. Instituto Nacional de Salud. Sistema de información del estado nutricional [Internet]. INS; 2014 [citado el 27 de marzo de 2016]. Disponible en: http:// www.portal.ins.gob.pe/es/cenan/ cenan-c2/vigilancia-alimentaria-ynutricional/sistema-de-informaciondel-estado-nutricional.

17. Banco Mundial. Prevalencia de anemia entre embarazadas [Internet]. BM; 2016 [citado el 11 de mayo de 2016]. Disponible en: http:// datos.bancomundial.org/indicador/ SH.PRG.ANEM.

18. Nwizu EN, Iliyasu Z, Ibrahim SA, Galadanci HS. Socio-demographic and maternal factors in anaemia in pregnancy at booking in Kano, northern Nigeria. Afr J Reprod Health. 2011 Dec;15(4):33-41.

19. Shamah-Levy T, Villalpando S, Rivera JA, Mejia-Rodriguez F, CamachoCisneros M, Monterrubio EA. Anemia in Mexican women: a public health problem. Salud Publica Mex. 2003;45 Suppl 4:S499-507.

20. Heidkamp RA, Ngnie-Teta I, Ayoya MA, Stoltzfus RJ, Mamadoultaibou A, Durandisse EB, et al. Predictors of anemia among haitian children aged 6 to 59 months and women of childbearing age and their implications for programming. Food Nutr Bull. 2013 Dec;34(4):462-79.

21. Bentley ME, Griffiths PL. The burden of anemia among women in India. Eur J Clin Nutr. 2003;57(1):52-60.
22. Kamruzzaman M, Rabbani MG, Saw A, Sayem MA, Hossain MG. Differentials in the prevalence of anemia among non-pregnant, ever-married women in Bangladesh: multilevel logistic regression analysis of data from the 2011 Bangladesh Demographic and Health Survey. BMC Women's Health. 2015 Jul 29;15:54. doi: 10.1186/ s12905-015-0211-4.

23. Tembhare A, Shelke S, Varma P, Tayade Sh, Tayade S. Socio-demographic determinants associated with iron deficiency anemia inpregnancy in rural population of central India. Int $\mathrm{J}$ of Biomed \& Adv Res. 2015;6(12):6.

24. Loza J, Dulanto A, Paz-Marchena A, Málaga G, Ticse R. [Differences in anemia detection in high altitude according to the World Health Organization]. Rev Peru Med Exp Salud Publica. 2012;29:157-8.

25. Laflamme EM. Maternal Hemoglobin Concentration and Pregnancy Outcome: A Study of the Effects of Elevation in El Alto, Bolivia. Mcgill J Med. 2011 Jun;13(1):47.

26. Gonzales GF, Tapia V, Gasco M, Carrillo C. [Maternal hemoglobin in Peru: regional differences and its association with adverse perinatal outcomes]. Rev Peru Med Exp Salud Publica. 2011;28(3):484-91.

27. Drake I, Bernztein R. Costobeneficio de un programa preventivo y terapéutico para reducir la deficiencia de hierro en Argentina. Rev Panam Salud Publica. 2009 Jan;25(1):39-46.

28. Miller JL. Iron deficiency anemia: a common and curable disease. Cold Spring Harb Perspect Med. 2013 Jul 1;3(7). pii: a011866. doi: 10.1101/ cshperspect.a011866.

Correspondencia: Akram Abdul Hernández Vásquez

Dirección: Calle Gavilán 3856, Ciudad Autónoma de Buenos Aires, Argentina Teléfono: (0054) 91122981572

Correo electrónico:akram.hernandez.v@upch.pe 